PROCEEDINGS OF THE

AMERICAN MATHEMATICAL SOCIETY

Volume 130, Number 1, Pages 253-258

S 0002-9939(01)06076-

Article electronically published on April 26, 2001

\title{
INCOMMENSURABILITY CRITERIA FOR KLEINIAN GROUPS
}

\author{
JAMES W. ANDERSON
}

(Communicated by Jozef Dodziuk)

\begin{abstract}
The purpose of this note is to present a criterion for an infinite collection of distinct hyperbolic 3-manifolds to be commensurably infinite. (Here, a collection of hyperbolic 3-manifolds is commensurably infinite if it contains representatives from infinitely many commensurability classes.) Namely, such a collection $\mathbf{M}$ is commensurably infinite if there is a uniform upper bound on the volumes of the manifolds in $\mathbf{M}$.

There is a related criterion for an infinite collection of distinct finitely generated Kleinian groups with non-empty domain of discontinuity to be commensurably infinite. (Here, a collection of Kleinian groups is commensurably infinite if it is infinite modulo the combined equivalence relations of commensurability and conjugacy in Isom ${ }^{+}\left(\mathbf{H}^{3}\right)$.) Namely, such a collection $\mathbf{G}$ is commensurably infinite if there is a uniform bound on the areas of the quotient surfaces of the groups in $\mathbf{G}$.
\end{abstract}

The purpose of this note is to explore conditions that imply that an infinite collection of distinct hyperbolic 3-manifolds is commensurably infinite. The main result, Theorem 0.1 is a criterion for a collection $\mathbf{M}$ of finite volume hyperbolic 3 -manifolds to be commensurably infinite. We also present a related criterion, Theorem 0.4 for a collection $\mathbf{G}$ of finitely generated Kleinian groups with nonempty domain of discontinuity to be commensurably infinite.

Recall that a Kleinian group is a discrete subgroup $\Gamma$ of the group $\operatorname{Isom}^{+}\left(\mathbf{H}^{3}\right)$ of orientation preserving isometries of hyperbolic 3-space. A hyperbolic 3-manifold is the quotient of $\mathbf{H}^{3}$ by a (torsion-free) Kleinian group. It follows from the rigidity theorems of Mostow 9] and Prasad [10 that if $N$ is a 3 -manifold that admits a finite volume hyperbolic structure, then the realization of its fundamental group as a Kleinian group is unique up to conjugacy in $\operatorname{Isom}^{+}\left(\mathbf{H}^{3}\right)$.

Two hyperbolic 3-manifolds, $N_{1}$ and $N_{2}$, are commensurable if they have a common finite cover, that is, if there exists a hyperbolic 3-manifold $N$ that is a finite cover of both $N_{1}$ and $N_{2}$. Commensurability is an equivalence relation on the set of hyperbolic 3-manifolds. A collection $\mathbf{M}$ of hyperbolic 3-manifolds is commensurably infinite if it contains representatives from infinitely many commensurability classes.

Two Kleinian groups $\Gamma_{1}$ and $\Gamma_{2}$ are commensurable if their intersection $\Gamma_{1} \cap \Gamma_{2}$ has finite index in both $\Gamma_{1}$ and $\Gamma_{2}$. This definition is a bit weaker than that of hyperbolic 3-manifolds (in that commensurable Kleinian groups give rise to commensurable hyperbolic 3-manifolds, but not necessarily vice versa), and so we need

Received by the editors May 18, 2000.

1991 Mathematics Subject Classification. Primary 57M50, 30F40; Secondary 20H10.

Key words and phrases. Kleinian group, hyperbolic 3-manifold, commensurable.

(C)2001 American Mathematical Society 
to adapt the definition of commensurably infinite in this case. Say that a collection $\mathbf{G}$ of Kleinian groups is commensurably infinite if it is infinite modulo the combined equivalence relations of commensurability and conjugacy in $\operatorname{Isom}^{+}\left(\mathbf{H}^{3}\right)$. Note that the combined equivalence relations of commensurability and conjugacy in $\operatorname{Isom}^{+}\left(\mathbf{H}^{3}\right)$ for Kleinian groups coincides with the equivalence relation of commensurability for hyperbolic 3 -manifolds.

Commensurability is a very natural equivalence relation to put on hyperbolic 3-manifolds and on Kleinian groups. We note here that there are several properties associated to hyperbolic 3-manifolds and Kleinian groups that are invariants of commensurability classes. For example, for a finite volume hyperbolic 3-manifold $N=\mathbf{H}^{3} / \Gamma$, the invariant trace field $\mathbf{Q}\left((\operatorname{tr}(\gamma))^{2} \mid \gamma \in \Gamma\right)$ is a commensurability invariant; see Reid [1].

Also, among torsion-free Kleinian groups, the property of having a subgroup that is locally free but not free is invariant under the combined equivalence relations of commensurability and conjugacy in $\operatorname{Isom}^{+}\left(\mathbf{H}^{3}\right)$. (Recall that a group is locally free if all its finitely generated subgroups are free.) This is an easy consequence of the result of Stallings [13] and Swan [14] that if $G$ is a torsion free group that contains a free subgroup $H$ of finite index, then $G$ is itself free.

The criterion for an infinite collection of finite volume hyperbolic 3-manifolds to be commensurably infinite is given in terms of a uniform bound on the volumes of the manifolds in the collection.

Theorem 0.1. Let $\mathbf{M}$ be an infinite collection of distinct finite volume hyperbolic 3-manifolds. Suppose there exists some constant $K>0$ so that $\operatorname{vol}(N) \leq K$ for all $N$ in $\mathbf{M}$. Then, $\mathbf{M}$ is commensurably infinite. Indeed, no commensurability class of manifolds in $\mathbf{M}$ is infinite.

Proof. We begin with a definition. Given a Kleinian group $\Gamma$, define its commensurability subgroup $\operatorname{comm}(\Gamma)$ in $\operatorname{Isom}^{+}\left(\mathbf{H}^{3}\right)$ to be

$$
\operatorname{comm}(\Gamma)=\left\{g \in \operatorname{Isom}^{+}\left(\mathbf{H}^{3}\right) \mid \Gamma \text { and } g \Gamma g^{-1} \text { are commensurable }\right\} .
$$

Note that $\Gamma \subset \operatorname{comm}(\Gamma)$. Moreover, if $\Gamma$ and $\Gamma^{\prime}$ are commensurable Kleinian groups, then $\operatorname{comm}(\Gamma)=\operatorname{comm}\left(\Gamma^{\prime}\right)$.

We argue by contradiction. Suppose there exist infinitely many manifolds $N_{n}=$ $\mathbf{H}^{3} / \Gamma_{n}, n \geq 1$, in $\mathbf{M}$ that are pairwise commensurable. Without loss of generality, we may choose the $\Gamma_{n}$ in their conjugacy classes in $\operatorname{Isom}^{+}\left(\mathbf{H}^{3}\right)$ so that they are pairwise commensurable as Kleinian groups. Since $\Gamma_{n}$ and $\Gamma_{m}$ are commensurable, we have that $\operatorname{comm}\left(\Gamma_{n}\right)=\operatorname{comm}\left(\Gamma_{m}\right)$ for all $n, m \geq 1$. Set $\Theta=\operatorname{comm}\left(\Gamma_{n}\right)$.

Although we do not give a precise definition here, we note that there exists a special class of Kleinian groups, the arithmetic Kleinian groups, which are roughly Kleinian groups defined by number theory. For a discussion of arithmeticity and some of its consequences, we refer the interested reader to the paper of Neumann and Reid [12] and to the references contained therein.

For the purposes of this note, it suffices to make use of a major result of Margulis 8] (see also the discussion in Zimmer [17]) that a finite co-volume Kleinian group $\Gamma$ is arithmetic if and only if $\Gamma$ has infinite index in $\operatorname{comm}(\Gamma)$. Note that arithmeticity of $\Gamma$ implies that $\operatorname{comm}(\Gamma)$ is not discrete, and hence is dense in $\operatorname{Isom}^{+}\left(\mathbf{H}^{3}\right)$.

We now apply a result of Borel [4], that given a constant $K>0$, there exist only finitely many arithmetic Kleinian groups $\Phi$ with $\operatorname{vol}\left(\mathbf{H}^{3} / \Phi\right) \leq K$. In particular, only finitely many of the $\Gamma_{n}$ can be arithmetic, and so there must exist some $j \geq 1$ 
for which $\Gamma_{j}$ is not arithmetic. In particular, $\Gamma_{j}$ is a subgroup of finite index in $\Theta=\operatorname{comm}\left(\Gamma_{j}\right)$, and so $\Theta$ is discrete and finitely generated. In particular, none of the $\Gamma_{n}$ are arithmetic.

By assumption, $\operatorname{vol}\left(\mathbf{H}^{3} / \Gamma_{n}\right) \leq K$ for all $n \geq 1$. Hence, the index of $\Gamma_{n}$ in $\Theta$ is bounded above by

$$
\left[\Theta: \Gamma_{n}\right]=\frac{\operatorname{vol}\left(\mathbf{H}^{3} / \Gamma_{n}\right)}{\operatorname{vol}\left(\mathbf{H}^{3} / \Theta\right)} \leq \frac{K}{\operatorname{vol}\left(\mathbf{H}^{3} / \Theta\right)}
$$

for all $n \geq 1$.

In particular, infinitely many of the $\Gamma_{n}$ have the same index in $\Theta$. Since $\Theta$ is finitely generated, it has only finitely many subgroups of each fixed finite index, and so infinitely many of the $\Gamma_{n}$ are equal, contradicting the assumption that the manifolds in $\mathbf{M}$ are distinct. This contradiction completes the proof.

Theorem 0.1 also holds, with the same hypotheses, for hyperbolic 2-manifolds, as the results of Margulis and Borel both apply to $\operatorname{Isom}^{+}\left(\mathbf{H}^{2}\right)$, as does the lower bound of the volume (in this case, area) of the quotient manifold (in this case, surface).

Also, for hyperbolic $n$-manifolds with $n \geq 4$, Theorem 0.1 is a trivial consequence of a result of Wang [16] that states that for each $K>0$, there are only finitely many isometry classes of hyperbolic $n$-manifolds of volume at most $K$.

One application of Theorem 0.1 is to consider the collection $\mathbf{M}_{n}$ of all finite volume hyperbolic 3 -manifolds with $n$ cusps.

Corollary 0.2. For $n \geq 0$, let $\mathbf{M}_{n}$ be the collection of all finite volume hyperbolic 3-manifolds with exactly $n$ cusps. Then, $\mathbf{M}_{n}$ is commensurably infinite.

Proof. This follows immediately from the properties of hyperbolic Dehn surgery. For a discussion of hyperbolic Dehn surgery, we refer the interested reader to Thurston [15], Gromov [6], or Benedetti and Petronio [2].

Let $N$ be a finite volume hyperbolic 3-manifold with $n+1$ cusps, and perform hyperbolic Dehn surgery on one of its cusps to obtain a collection of finite hyperbolic 3-manifolds with $n$ cusps; that is, a subcollection of $\mathbf{M}_{n}$. The volumes of the surgered manifolds are bounded above by $\operatorname{vol}(N)$, and the surgered manifolds are distinct, as their volumes increase to $\operatorname{vol}(N)$. By Theorem 0.1 the collection of surgered manifolds is commensurably infinite, and so the larger collection $\mathbf{M}_{n}$ is also commensurably infinite.

We note that, with this proof, we are unable to obtain the slightly stronger conclusion that no commensurability class of manifolds in $\mathbf{M}_{n}$ is infinite.

Corollary 0.2 in the case of $n=0$ is due to Maclachlan and Reid [7] using arithmetic techniques. Also, Reid [11] has shown that the collection of closed hyperbolic 3-manifolds fibering over the circle is commensurably infinite. However, it does not seem that the above corollary for $n$-cusped hyperbolic 3-manifolds exists in the literature.

Another corollary of Theorem 0.1, and one that also makes use of the machinery of hyperbolic Dehn surgery, involves a bound on the rank of the fundamental group.

Corollary 0.3. Let $\mathbf{N}_{2}$ be the collection of all closed hyperbolic 3-manifolds whose fundamental groups are generated by exactly two elements. Then, $\mathbf{N}_{2}$ is commensurably infinite. 
Proof. Let $N$ be a finite volume hyperbolic 3-manifold that has one cusp and whose fundamental group is generated by two elements; for example, we may take $N$ to be the complement of the figure 8 knot in the 3 -sphere $\mathbf{S}^{3}$. Consider all the closed hyperbolic 3-manifolds obtained by performing hyperbolic Dehn surgery on $N$.

As in the proof of Corollary 0.2 the collection of surgered manifolds gives an infinite subcollection of closed hyperbolic 3-manifolds whose volumes are bounded by $\operatorname{vol}(N)$. To see that each surgered manifold $P$ has a 2 -generator fundamental group, note that since $P$ is obtained from $N$ by hyperbolic Dehn surgery, there is a homomorphism from the fundamental group of $N$ to the fundamental group of $P$. Since $N$ has a 2-generator fundamental group and since the fundamental group of $P$ cannot be a 1-generator group, we see that each of the surgered manifolds has a 2-generator fundamental group, and so all the surgered manifolds are contained in $\mathbf{N}_{2}$. Applying Theorem 0.1 yields the desired result.

It may be that there are analogues of Corollary 0.3 for hyperbolic 3 -manifolds with $n$ generator fundamental groups for $n \geq 3$, but these would seem to require a level of analysis of the operation of hyperbolic Dehn surgery that is beyond the scope of the present work.

We close this note with the analogue of Theorem 0.1 for finitely generated Kleinian groups with non-empty domain of discontinuity.

Recall that the action of $\operatorname{Isom}^{+}\left(\mathbf{H}^{3}\right)$ on $\mathbf{H}^{3}$ by isometries extends to an action of $\operatorname{Isom}^{+}\left(\mathbf{H}^{3}\right)$ on the Riemann sphere $\overline{\mathbf{C}}$, viewed as the sphere at infinity of $\mathbf{H}^{3}$, by conformal homeomorphisms. Given a Kleinian group $\Gamma$, define the domain of discontinuity $\Omega(\Gamma)$ of $\Gamma$ to be the largest open subset of $\overline{\mathbf{C}}$ on which $\Gamma$ acts properly discontinuously, and define the limit set $\Lambda(\Gamma)$ of $\Gamma$ to be the complement of $\Omega(\Gamma)$ in $\overline{\mathbf{C}}$.

A Kleinian group that is not virtually abelian is non-elementary. When $\Gamma$ is nonelementary, its limit set is infinite, and so there is a canonical metric of constant curvature -1 on $\Omega(\Gamma)$, the Poincaré metric, so that $\Gamma$ acts on $\Omega(\Gamma)$ as isometries of the Poincaré metric. It is a fundamental result of Ahlfors 1 that if $\Gamma$ is finitely generated (and non-elementary), then the area of the quotient $\Omega(\Gamma) / \Gamma$ (calculated with respect to the Poincaré metric) is finite.

Theorem 0.4. Let $\mathbf{G}$ be an infinite collection of pairwise non-conjugate, nonelementary, finitely generated Kleinian groups with non-empty domain of discontinuity. Suppose there exists a constant $K>0$ so that area $(\Omega(\Gamma) / \Gamma) \leq K$ for all $\Gamma$ in G. Then, $\mathbf{G}$ is commensurably infinite. Indeed, no class in $\mathbf{G}$ modulo the combined equivalence relations of commensurability and conjugacy in $\operatorname{Isom}^{+}\left(\mathbf{H}^{3}\right)$ is infinite.

Proof. As in the proof of Theorem [0.1, we argue by contradiction. Suppose there are infinitely many groups $\Gamma_{1}, \Gamma_{2}, \ldots$ in $\mathbf{G}$ that are pairwise equivalent under the combined equivalence relations of commensurability and conjugacy in $\operatorname{Isom}^{+}\left(\mathbf{H}^{3}\right)$. By taking appropriate conjugates and by noting that the limit sets of commensurable Kleinian groups are equal, we may assume without loss of generality that the limits sets $\Lambda\left(\Gamma_{1}\right), \Lambda\left(\Gamma_{2}\right), \ldots$ of the $\Gamma_{n}$ are all equal.

It is well known (see for instance Greenberg [5] ) that the stabilizer in $\operatorname{Isom}^{+}\left(\mathbf{H}^{3}\right)$ of the limit set of a non-elementary finitely generated Kleinian group $\Gamma$ is a finite extension of $\Gamma$, and hence is itself a finitely generated Kleinian group, unless the limit set $\Lambda(\Gamma)$ is a round circle in $\overline{\mathbf{C}}$. 
In the case that the limit set of one (and hence all) of the groups in $\mathbf{G}$ is a round circle, then all the groups in $\mathbf{G}$ (or an index two subgroup of each) are actually contained in (a conjugate of) $\mathrm{Isom}^{+}\left(\mathbf{H}^{2}\right)$ (in Isom ${ }^{+}\left(\mathbf{H}^{3}\right)$ ). Since there is a uniform upper bound on the areas of the quotients, this case falls under the aegis of Theorem 0.1, and particularly the remark following its proof. Hence, we can assume that none of the groups in $\mathbf{G}$ have limit set a circle in $\overline{\mathbf{C}}$.

Let $\Theta$ be the stabilizer in $\operatorname{Isom}^{+}\left(\mathbf{H}^{3}\right)$ of $\Lambda\left(\Gamma_{1}\right)$. (In fact, $\Theta=\operatorname{comm}\left(\Gamma_{1}\right)$.) Then, all the groups $\Gamma_{1}, \Gamma_{2}, \ldots$ are subgroups of $\Theta$. Since there is a universal lower bound on the possible area of the quotient surface of a finitely generated Kleinian group, namely $\frac{\pi}{21}$, the indices $\left[\Theta: \Gamma_{n}\right]$ are all bounded above by

$$
\left[\Theta: \Gamma_{n}\right]=\frac{\operatorname{area}\left(\Omega\left(\Gamma_{n}\right) / \Gamma_{n}\right)}{\operatorname{area}(\Omega(\Theta) / \Theta)} \leq \frac{21 K}{\pi} .
$$

As in the proof of Theorem 0.1 , this forces infinitely many of the $\Gamma_{n}$ to be equal, contradicting the assumption that the $\Gamma_{n}$ are distinct. This contradiction completes the proof.

There is a corollary to Theorem 0.4 that is analogous to Corollary 0.3 of Theorem 0.1 .

Corollary 0.5. Given $n \geq 2$, let $\mathbf{G}_{n}$ be an infinite collection of pairwise nonconjugate, non-elementary Kleinian groups with non-empty domain of discontinuity generated by $n$ elements. Then, $\mathbf{G}_{n}$ is commensurably infinite. Indeed, no class in $\mathbf{G}_{n}$ modulo the combined equivalence relations of commensurability and conjugacy in $\operatorname{Isom}^{+}\left(\mathbf{H}^{3}\right)$ is infinite.

Proof. The proof is an immediate consequence of Bers' refinement of Ahlfors' theorem. Bers' theorem [3] states that if $\Gamma$ is an $n$-generator non-elementary Kleinian group with non-empty domain of discontinuity, then $\operatorname{area}(\Omega(\Gamma) / \Gamma) \leq 4 \pi(n-1)$. Since there is a uniform bound on the areas of the quotient surfaces of the groups in $\mathbf{G}_{n}$, the corollary follows immediately from Theorem 0.4 .

I would like to thank the referee for a careful reading of this note and for the contribution of a number of suggestions that have greatly improved the clarity of the exposition.

\section{REFERENCES}

[1] L. V. Ahlfors, 'Finitely generated Kleinian groups', Am. J. Math. 86 (1964), 413-429.

[2] R. Benedetti and C. Petronio, Lectures on Hyperbolic Geometry, Springer-Verlag Universitext, 1992. MR 94e:57015

[3] L. Bers, 'Inequalities for finitely generated Kleinian groups', J. Analyse Math. 18 (1967), 23-41. MR 37:5383

[4] A. Borel, 'Commensurability classes and volumes of hyperbolic 3-manifolds', Ann. Scuola Norm. Sup. Pisa Cl. Sci. (4) 8 (1981), pp. 1-33. MR 82j:22008

[5] L. Greenberg, 'Commensurable groups of Moebius transformations', in Discontinuous groups and Riemann surfaces, ed. by L. Greenberg, Annals of Mathematics Studies 79, Princeton University Press, Princeton, 1974, 227-237.

[6] M. Gromov, 'Hyperbolic manifolds according to Jørgensen and Thurston', Séminaire Bourbaki $32^{e}$ année (1979-80), Lecture Notes in Mathematics 842, Springer-Verlag, pp. 1-14.

[7] C. Maclachlan and A. W. Reid, 'Commensurability classes of arithmetic Kleinian groups and their Fuchsian subgroups, Math. Proc. Cam. Phil. Soc. 102 (1987), 251-257. MR 88j:20040

[8] G. A. Margulis, Discrete Subgroups of Semisimple Lie Groups, Ergebnisse der Mathematik und ihrer Grenzgebiete 17, Springer-Verlag, New York, 1991. MR 92h:22021 
[9] G. D. Mostow, Strong rigidity of locally symmetric spaces, Annals of Mathematics Studies 78, Princeton University Press, Princeton, 1973. MR 52:5874

[10] G. Prasad, 'Strong rigidity in Q-rank 1 lattices', Invent. Math. 21 (1973), 255-286. MR 52:5875

[11] A. W. Reid, 'A note of trace-fields of Kleinian groups', Bulletin L. M. S. 22 (1990), 349-352.

[12] W. D. Neumann and A. W. Reid, 'Arithmetic of hyperbolic manifolds', Topology '90 (Columbus, OH, 1990), Ohio State Univ. Math. Res. Inst. Publ. 1, de Gruyter, Berlin, 1992, 273-310. MR 94c:57024

[13] J. R. Stallings, 'On torsion-free groups with infinitely many ends', Annals of Math. (2) 88 (1968), pp. 312-334. MR 37:4153

[14] R. G. Swan, 'Groups of cohomological dimension one', J. Algebra 12 (1969), pp. 585-610. MR 39:1531

[15] W. P. Thurston, The geometry and topology of 3-manifolds, lecture notes, Princeton University, 1978.

[16] H. C. Wang, 'Topics in totally discontinuous groups', in Symmetric spaces, edited by W. M. Boothby and G. L. Weiss, Pure and Applied Mathematics 8, Marcel Dekker, Inc., New York, 1972, 460-485.

[17] R. J. Zimmer, Ergodic Theory and Semisimple Groups, Monographs in Mathematics 81, Birkhäuser, Boston, 1984. MR 86j:22012

Faculty of Mathematical Studies, University of Southampton, Southampton SO17 1BJ, ENGLAND

E-mail address: j.w.anderson@maths.soton.ac.uk 